\title{
Why do we need research?
}

\author{
Bob McKee \\ Chief Executive of CILIP
}

A lightly edited transcript of the paper given to LIRG on 4 July 2003 as part of the CILIP Umbrella 2003 Conference

4 July 2003

I don't think of myself as inhabiting the research community. And yet, on reflection, I have had a sort of on/off relationship with research throughout my career. Indeed I first became a library assistant in order to finance my "other life" as a doctoral research student at the University of Birmingham's Shakespeare Institute. And, at a key transitional point in my career I returned to academic life - including research - at the then library school at what is now the University of Central England.

So I've been involved in the world of research from two different academic perspectives. And I've also been involved - I suppose over quite a long time now - in aspects of research policymaking and programme delivery. As a former member (quite a while ago now) of the LISU Advisory Committee. As a member (in the mid 1990s) of the Steering Group for the Aslib Review of PL Service - described by Roger Bowes (with characteristic understatement) as "the largest piece of research into public libraries ever undertaken in the world". As a member (throughout its life) of the LIC's Research Committee. And, more recently, as a member of the selection panel for the BL Cooperation and Partnership Programme.

And, of course, my present role with CILIP involves connections with the research community because CILIP undertakes a number of activities related to research - about which more later.

So I've seen research from a number of perspectives in the course of my career - but I've not yet mentioned the perspective that is the most fundamental in shaping my views on the research agenda.

The literature of research in our domain talks a lot about the importance of the "practitionerresearcher" - as it should do in an academic domain which is clearly part of Professional and Vocational Education - and this is often linked to the concept of the "reflective practitioner" which underpins CILIP's Chartering process and will underpin CILIP's future process of accredited continuing professional development.

Well, I've always sought to be a "reflective practitioner" and at times I've also been a "practitioner-researcher". I've always believed in that continuous process of iteration between research and practice.

So for example, when I reflect on my work in the 1970s in Birmingham's inner city in the early days of community librarianship I can see a continuous iterative process of research, reflection, practice, policy which connects directly from the work in the 1970s into the current work on Framework for the Future - just as there's a sort of "audit trail" of research 
and practice linking current work on social inclusion to work in the 1970s on community development.

And similarly when I reflect on my work in the early 1980s in further education (in the early days of resource based learning and student centred learning) I see again a clear connection expressed through a continuous process of iteration between research and practice - with today's education agenda around learning styles, learning environments, widening participation, lifelong learning, learning support and so on.

Indeed it is research in this field - particularly interdisciplinary research into the sociology and psychology of the learning process - which is currently informing my thinking about the future of CILIP Groups as communities of practice within our broad professional domain and our broad framework of education, training and professional development.

And then perhaps, for me, the most vivid example of this longitudinal perspective on the iteration between research and practice comes with technology and the application of new technology to library service.

Some of the older folk amongst you may remember the government initiative "IT82" designed to promote the opportunities of information technology - and the programme of largescale action research projects (funded through DTI and the emerging IT industry) which followed on from IT82.

I was involved in one such project in 1983/84 using the technology of Viewdata (sometimes called videotex; best known brand name being BT's "Prestel" service) to explore the uses of networked information and interactive services in domestic, business and educational contexts. And there's certainly a very clear audit trail of research and development connecting those early rudimentary action research projects into Viewdata in the early 1980s with today's digital agenda, today's ICT industry, today's focus on e-commerce, e-learning and e-government.

Similarly there's a clear connection between the research that was done as part of the unsuccessful LIC/LA Millennium Libraries bid - research which developed a financial and technical model for a network infrastructure connecting all public libraries - and the later successful bid to develop the People's Network.

Just as there's a clear connection between the findings of BLRDD funded IT projects in public libraries in the mid 1990s (like IT Point in Solihull and CLIP in Croydon) and the recent findings written up by Peter Brophy about early patterns of use of the People's Network.

What this longitudinal reflection (into these three areas of practice around community librarianship, lifelong learning and technological development) brings out is, I think, something quite encouraging.

At any one moment in time we might find causes for frustration with the research agenda. Frustration over funding. Frustration over policy. Frustration over the apparent disconnection between research and practice. But when you take the long view - as I've just tried to do, based on my own experiences - there is evidence that research does transfer over time into practice and that the iteration between research, reflection, practice and policy does 
take our domain forward both in terms of our knowledge base and in terms of our professional practice.

But why is all this important? To quote the title of the talk - Why do we need research? And indeed - what do we mean by "research"?

When I did my $\mathrm{PhD}$ in the mid 1970s research was defined as "an original contribution to knowledge" and was (at least in the academic environment I then inhabited) very much set in the world of scholarship with the purpose of deepening understanding within the academic discipline rather than connecting with or contributing to the world of practice. So researching into Elizabethan drama at the Shakespeare Institute at that time was about scholarship - not about stagecraft or acting or the current practice of drama in the theatre. Although this has now changed and there is now a very close interrelationship between the academic work of the Shakespeare Institute and the practical work of the Royal Shakespeare Company and other current manifestations of Shakespeare in practice both on stage and on film.

So this suggests at least two types of research:

- research which has validity within the academic environment - which contributes to the knowledge base

- research which has value within the practitioner environment - which contributes to service innovation and service development

and you can see quite often that the literature (certainly in our domain) shows different definitions of research depending on these two different perspectives - of the academic researcher and the practitioner researcher.

And then there's a third type of research which at present seems to be the dominant variety of the species - research to inform policy.

This is very much the approach taken by Resource. The role of research so far as Resource is concerned seems to be essentially about helping to develop evidence-based policy to inform the Resource work programme. While I was preparing this paper, Resource very helpfully sent me a note of its current research activities. As the note says, "Details of research projects commissioned by Resource tend to appear with details of specific work programmes rather than being pulled together in any one place". And the note goes on to outline research underpinning Resource's work related to: statistical evidence gathering; learning and access; workforce development; standards and guidelines; the People's Network; international work; and work on collection management.

The note also says that the published Resource Research Strategy is deemed to be out of date - and that a process is now beginning to revise the Research Strategy as part of Resource's Strategic Planning for the forthcoming period 2004-2007.

I must say that this type of research to inform evidence-based policymaking seems to be prevalent everywhere at present - including CILIP.

Our position paper, published last year, on National Information Policy is underpinned by research - funded by Resource and undertaken by a team at Loughborough University - into developments worldwide on NIP. 
Our advocacy paper Start with the child is based on a major research project - again funded by Resource - which examined the needs, motivation and attitudes of children and young people.

This evidence-based approach is also seen in policy papers from government.

The recent paper from LISC (Wales) on Mapping social inclusion in publicly funded libraries in Wales and the recently published Tomorrow's libraries report on the public library service in Northern Ireland both include chapters explicitly on methodology: with the policy work in Wales underpinned by surveys and interviews; and the policy work in Northern Ireland underpinned by a very substantial research programme of desk research and focus groups and surveys and submissions, and a major "futureSearch" conference (in which I participated), and a range of Action Planning Groups.

And as well as this research-based work in Wales and Northern Ireland we have, in England, Framework for the future, a document which is again based on research and analysis carried out by the policy think tank, Demos.

Much of this policy-related research, you'll note in passing, is not being carried out by what might be termed the traditional LIS research community but by independent research/consultancy agencies. And some people might not even include this type of work in their definition of research. But I would - and I know that LIRG also would, given LIRG's mission to foster links between research and practice and (by extension) between research and policy.

I take a broad view - an inclusive view if you like - of research and I would include what some might describe as consultancy or as commentary or as developmental activity, all under the broad "umbrella" of research.

For me, research is an enquiry which is soundly based (in terms of its methodology) and which adds something of value (in terms of its content) to our knowledge base or to our professional practice or to our policy development. So if it has rigour and it has relevance then that's all it needs for me in order to be described as "research".

But why do we need research as so defined? I would submit three reasons:

- to increase our knowledge and understanding (and part of that, of course, is about research-based teaching by LIS educators)

- to provide the evidence to underpin and inform policy-making and decision-making

- and to advance professional practice and underpin service development by the generation and testing of new ideas which can then lead to service innovation and improvement

And if that three-part justification for LIS research sounds familiar it's not surprising because it's taken almost word-for-word from the study commissioned by CILIP and recently published by the Centre for Information Research at UCE into The LIS research landscape.

That research was commissioned, at my instigation, because of what might be described as prevailing concerns about the current state of research within the LIS community. 
So what I'd like to do now is to track back once again and take another longitudinal perspective - but switch the methodology so that this time the findings are based, not on personal reflection but on reading the literature - in order to draw out the key issues which have emerged, over time, around the topic of research within the LIS domain in the UK. The issues which have formed and which continue to form the dominant features of our research landscape.

My starting point for this literature review was that sequence of publications (that used to exist) which reviewed the last five years of library and information work in the UK - a sequence which ended (so far as I can detect) with the volume bringing us up to 1990.

Each volume had a chapter on research and I traced the 15-year period from the start of my career in the mid 1970s to 1990.

The tone of the reports on research in the ten year period 1976-85 is quite positive - and Stephen Roberts looking back on that period (in writing his review of 1986-90) calls it "a period of blooming and fruiting", reaping the benefits of previous investment in and development of the LIS research infrastructure.

There was growth in the volume of research, maturity and stability in the machinery (the organisation and administration) of research. There was a strong research community providing continuity and an accumulation of experience and expertise. There was movement of individuals between practice and research bringing those two communities of interest closer together. There was the central role (the anchor role or catalytic role) of BLRDD providing funding for research, determining strategic priorities for research, supporting innovation (especially technological innovation) and thereby service development.

And the conclusion by Nick Moore (of Acumen) who was reviewing the period 1981-85 was that "the [LIS] research machine in Britain is well established and functioning quite effectively" although Nick did add that for him "the test remains the extent to which practitioners and others take up and act on the results of the research process".

Well, it's always easy (in a sort of warm and sentimental way) to hark back to a supposed "golden age" - and Stephen Roberts, looking back in his usual forthright manner a few years later, did feel that Nick's view had been "a little too complacent".

But Nick did identify a number of issues which were of concern even in that "golden age" of growth and relative stability - issues which remain matters of concern today.

There was a great deal of "in-house" research going on (research carried out within particular library services) but not much of it was being harvested and made available to the wider LIS community. I think this is still true - and this links to another perennial issue raised by Nick: the importance of dissemination. As that wise man Maurice Line once said - if research is going to have any impact it has first to be known about.

This issue of dissemination is in turn intimately connected with the other issue raised by Nick - that of transferring research into practice. The issue at the heart of LIRG's mission. 
David Streatfield put together a very useful piece of research for the LIC (published in 2000) called Metamorphosis: transferring research into practice which reviews a number of professional domains related to (but separate from ) the LIS domain - health, social care, education. There's a lot to learn from those other domains about this issue of transferring research into practice - about partnership, about behavioural change, about (again) the concept of the "reflective practitioner".

But the central point made by David and implied in Stephen Roberts' review is that researchers and practitioners tend to inhabit different communication worlds. The process of reporting research in progress or research findings for the purposes of academic peer review and the Research Assessment Exercise is very different from the process of reporting research activity to the practitioner. David makes the point - in the context of education but it's equally applicable to LIS - that researchers need to see communication with practitioners as a "high status" activity; just as practitioners need to see the act of interpreting evidence developed through research as a "high status" activity central to reflective practice. So some perennial problems. Of transferring research into practice. Of dissemination. Of harvesting "in house" research activity.

And the other point to make when looking back on these reviews of research activity in the 70 s and 80 s is how inward facing they now seem. The focus is the library and information domain - not (as it would surely be today) LIS set into the context of the bigger picture: the social, educational, economic and cultural health of the nation as the Resource WILIP project rather grandly puts it. And the focus of these past reviews was also very much the UK there's not much sense of an international research community. And, I would hope that's also now changing given the global nature of the information society and the way in which issues of professional interest and concern across the LIS domain transcend national and cultural boundaries.

Stephen Roberts begins his review of the five year period to 1990 by referring to an event which some of you may well remember - the conference on Research policy in librarianship and information science held just down the road in Salford in 1990, organised by LIRG, funded by BLRDD, with the proceedings edited by Colin Harris and published by Taylor Graham.

If you take Stephen's review and the 1990 Salford Conference proceedings together, a number of further themes emerge which continue to feature in today's research landscape.

Stephen's conclusion was "there is a lively and active research scene in UK LIS but it is under-resourced and faces problems of identity and structure".

I think that would be seen as fair comment today. Yes, problems with funding, identity and structure - but there is lively and active professional discourse underpinned by research and innovation. You can see it in the sessions at this Umbrella Conference and in the feature articles carried by Update and in the books published by Facet Publishing and in the rich mix of seminars and events organised across our profession. There is a sense of lively professional discourse and debate and development across the LIS domain. So - looked at in these terms - we do have "a lively and active research scene".

And we also have today the three trends detected by Stephen in his review. One trend being the broadening of the domain so that - as he put it - "field boundaries are being redefined". 
He noted that LIS researchers were now getting funding from, for example, the Economic and Social Research Council and the Science and Engineering Research Council. Another trend being a move away from what Stephen called "scholarly, blue-sky, open-ended projects" to what he called "strategic" projects - that is the move towards research to inform policy, research to inform practice, which I've already mentioned. And the other trend being a decline in research-related investment leading to what Stephen described as "the inability to sustain a thriving research culture".

And all the issues raised by Nick Moore and by Stephen Roberts came up for consideration at the 1990 Salford Conference - and remain, I think, central concerns for the LIS research community today.

I was at the Salford Conference and in my paper to that Conference I talked about the different perspectives on research from the academic community and from the practitioner community - a difference I've already mentioned in this talk.

At Salford I talked about the barriers which got in the way (and still get in the way) of practitioners engaging in research activity. Lack of time, lack of support from employers, lack of in-house skills and experience, the complexities attached to the process of seeking research funding, the perception of some research funding bodies as impersonal and bureaucratic, the lack of a critical mass of practitioner-researchers.

I also talked about the inward focus of much LIS research as opposed to the need, strategically, to engage LIS in this wider world of social, educational, cultural, economic agendas - and I suppose in my stance at the time and Stephen Roberts' stance at the time you can see again something of that tension between the researcher working in the academic context and the practitioner (by which by then I suppose I meant the senior practitioner) working in what is an essentially political context of policy making and strategic resource allocation.

The keynote paper at the Salford Conference was given by Maurice Line (who has always of course supported the concept of the "practitioner-researcher") and Maurice asked, in typical manner, a question which was to become highly pertinent as we moved through the 1990s from the era of BLRDD to the era of the LIC - should research (as it impacts on publicly funded bodies) [asked Maurice] be funded through government or a government agency; and if so should it be coordinated around some sort of nationally agreed agenda? Should research projects therefore be largely solicited rather than being unsolicited?

Because there was a time in that "golden age" of BLRDD when the academic community seemed to drive the research agenda. When BLRDD issued open calls and was hospitable to unsolicited approaches. I know: I benefited.

But the later 1990s and particularly the work of the LIC marked a transition to the point where the policy-making community seemed to set the research agenda, where calls for proposals were very clearly scoped and focused, and where most research within the LIS domain was solicited rather than unsolicited. And I suppose the current stance of Resource (in terms of commissioning research only to inform policy and develop practice) is a logical extension of that transition from the traditional BLRDD position to the more recent LIC position. 
The task of drawing out the conclusions and recommendation which emerged from the 1990 Salford Conference fell to Lynne Brindley, and what she said then resonates absolutely, it seems to me, with the current research landscape. So I paraphrase here Lynne's conclusions:

$>$ There is a multiplicity of funding bodies and better guidance to funding opportunities is needed

$>$ There needs to be a national policy for LIS research but it's not clear who should develop it

$>$ There appear to be major gaps between the needs of practitioners and the needs of researchers and something must be done to bridge the gap

$>$ LIS research is relatively small scale and inward looking. We need to develop strategic alliances linking LIS to the Bigger Picture

$>$ There are concerns about the development of a sufficient body of researchers within the LIS community and there is particular concern about the arrangements for bursaries, research studentships, postdoctoral scholarships and so on

$>$ And more needs to be done to evaluate the impact of research - and to disseminate and exploit research

And to Lynne's comments I would add a further point made during discussion at the Salford Conference by Tom Wilson - that you can only develop research competencies if you have continuity of research funding: a point that became one of deep concern to the LIS research community around 3-4 years ago when the decision was taken to "freeze" LIC research activity as part of the transition from the LIC to Resource.

I think the 1990 Salford Conference set an agenda which was then some years later taken forward by the LIC Research Committee when the LIC was established in 1995; and that agenda remains relevant today particularly because it could be argued that the gains made by the LIC in the area of research appear to have been lost in the early years of Resource although I have hopes that those gains may be regained as Resource becomes a more mature organisation and revisits its Research Strategy.

You know the recent history. The LIC established a Research Committee (chaired by Mel Collier). The Committee set out to develop a nationally coordinated strategic approach to research, development and innovation for the LIS domain. There was a substantial mapping exercise, a substantial consultation exercise, a draft document sent out for further consultation in 1997 and then in March 1998 publication by the LIC of Prospects: a strategy for action. This report addressed two clusters of issues - the agenda for research content and the machinery of research infrastructure.

A nationally coordinated programme of research was proposed around five themes:

- issues around access and connectivity

- issues around content and resource management

- issues around skills and competencies

- issues around the economics of LIS

- issues around the value and impact of LIS

And five infrastructural issues were also put forward as needing attention: 
- the need for a strategic and coordinated approach to the forward planning of research activity

- the need for mapping and guidance around the funding of research

- the need for a stronger skill base and better evaluation processes to improve the quality (and the quality assurance) of research

- the need for more effective means of communication about research activity and research findings

- the need to learn more about the process of transferring research into practice so that this might become a more effective/widespread process

Prospects having set the agenda, LIC then began to move into the delivery phase with the integration of what had been BLRDD (what was now BLRIC) into the LIC.

And with these additional resources transferred from the BL, the LIC published a Research Plan for 1999-2002 and issued the first two calls for proposals, one fairly general, one quite precisely targeted - and everything seemed set fair: until this renewed momentum of LIS research got caught up in the dis-establishment of the LIC and the establishment of Resource - at which point the agenda set out by the LIC (and the LIS community) in Prospects was superseded, so far as Resource was concerned, by the report by Professor John Shepherd into Research priorities and practices for the new Museums, Libraries and Archives Council.

I think it's fair to say that the clumsy way in which the transition from the LIC to Resource was handled generally destabilised the LIS research community:

- the progress made through the LIC appeared to have been lost

- the funding for research which had transferred from the BL to the LIC and then to Resource appeared to have been redirected

- the future for established strands of research activity appeared uncertain as the BNB Research Fund was discontinued and the funding of research centres like LISU was made subject to annual review

And there was - and I think still is - a serious concern that the research community within the LIS domain and the (fairly fragile) research culture within the LIS domain would be seriously damaged by the discontinuity and lack of apparent concern for the research agenda by Resource. At the heart of our research community had always been (at least in our memories) a dedicated LIS locus for research - first BLRDD then BLRIC then LIC. Resource had taken this away. And left in its place a vacuum.

And it was in this context - of discontinuity and concern and a vacuum - that we at the then LA decided to commission our review of the current state of the LIS research landscape.

We commissioned the Centre for Information Research at UCE to examine the research landscape for the LIS domain in the UK with research defined (as I've done throughout this talk) in its broadest sense.

The study (carried out by Clare Nankivell and Sarah McNicol) has now been published and is soon to be considered by CILIP's Policy Development Committee. You may have seen a press release we issued in June highlighting the study which talks about: a research agenda that lacks direction; decreased opportunities for LIS research following the demise of the LIC; funding being more difficult to obtain with the fragmentation of funding sources and 
less dedicated library and information funding; a consequent lack of research in key areas principally in what used to be called the "special" or "workplace" library sector and also, of course, a lack of long term so-called blue sky research not directly related to current policy concerns.

Previous recent studies have made similar points. BAILER's stated view is that the amount of funding available for research within the LIS domain is insufficient to

- foster the research capabilities of newer staff and so develop a critical mass of research expertise

- ensure that research is of high quality

- establish a basis for a "research into practice" culture (such as we see emerging in health or education or social care)

And this supports the need expressed by David Haynes, David Streatfield and Noeleen Cookman in their Review of research funding for LIS (LIC 2002) that the LIS domain in the UK at present lacks a research culture and research credibility.

It also reinforces the point made by Judith Elkin in the Overview report on the 2001 Research Assessment Exercise for Library and Information Management - that much research in the LIS domain is of low quality.

It is interesting and somewhat concerning to reflect that when the AHRB become the AHRC our domain might find it difficult to match the presumably higher standards required for Research Council funding.

For example AHRB wants to move towards support for doctoral rather than Master's studies because a Research Council is about supporting research and doctoral research is important and this may not work to our advantage.

There has been a decline in the numbers of research awards in the LIS domain but AHRB sees itself as quality driven and wants to move to a common set of (high) standards with very little ring fencing for funding for particular subject areas.

I have some sympathy with this view and I will need persuading if I am to support any ring fencing of subject areas - including our own academic domain.

AHRB is to produce a new strategic plan to run over the next five years and claims that it wishes to develop closer relationships between itself and key domains within its broad area of activity. And I would hope that CILIP (and by extension LIRG) will be very much part of that closer relationship.

So. It can be seen as a difficult and a frustrating time for the LIS research community and for all those of us who believe that we need a strong research capability and a strong research culture to deepen our knowledge base, inform our policy making, develop our professional practice and support service innovation.

So. Where do we see positive signs? And how do we go forward? 
Well, for me the most positive sign is a renewed willingness to engage with the research agenda. I'm an habitual optimist but I do detect signs that Resource as it matures as an organisation is beginning to revisit the issue of research - and this is reinforced by the news that Resource is to review its research strategy.

And in a partnership between Resource and the British Library and BAILER and the AHRB and other research-related stakeholders and, of course, CILIP (including LIRG) there may be a positive way forward which enables the LIS domain:

- to redefine our research agenda and revisit the issue of a cohesive LIS research strategy

- to rebuild our research capacity and become a more research-oriented community of practice with more collaboration and iteration between practitioner and researchers

- to engage with the wider research community as our profession continues to look outward to social, educational, cultural and economic environments - with more focus on interdisciplinary research and in consequence, more access perhaps to Research Council funding

- and to improve our processes of research journalism and the transfer of research into practice

In terms of a cohesive strategy for LIS research - a strategy to give direction to our research agenda - I do believe that this could be put together quite quickly.

We know what the infrastructural issues are because most of them are longstanding issues as I've made clear by their recurrence in this talk.

And we know what the priorities for research activity are. Or, at least, we can arrive at them fairly quickly by a process of triangulation - bringing together: the research programme set out by the LIC in Prospects which I believe still stands up to scrutiny; the current range of research topics identified in our research into the current LIS research landscape; and the research agenda emerging from the work of Resource.

The work being done by Resource on Workforce Development, on issues around access and learning, and on "wider information and library issues" (WILIP) and by the Regional Agencies established by Resource across England could be brought together to form a coherent research agenda - and I understand something along these lines is happening via Resource's Strategic Planning for the period 2004-2007.

With a new Chair and new Board members there is I believe a new willingness within Resource to engage with these issues.

And then there's CILIP. The landscaping study identifies a number of roles for CILIP:

- in terms of research foresight and horizon scanning

- in terms of ensuring that the voice of research is heard by government and other key stakeholders

- in terms of brokering research relationships across the library/information/knowledge continuum

- in terms of promoting evidence-based practice and evidence-based policy making 
- and in terms of encouraging research skills through our qualifications framework and our emerging framework of continuing professional development

Similarly the very important report on CILIP in the knowledge economy (published last year and developed by a high powered group of information professionals chaired by Nigel Oxbrow) sets out a role for CILIP in information research and development - and there is total synergy between this role (as identified by a group of information practitioners) and the role as outlined in the landscaping study (produced largely from an academic perspective).

The academic community and the practitioner community agree on CILIP's role with regard to research.

So. There is a clear agenda and a clear role for CILIP within that agenda. And LIRG has a very important role to play in this because of LIRG's mission to link together research and practice. We need to work hard to encourage CILIP Members to join LIRG as a CILIP Special Interest Group, just as we need to encourage our colleagues across the profession to develop a culture of "research in practice".

So. By way of conclusion let me return to Stephen Roberts and his review of the LIS research landscape around the time of that 1990 Salford Conference.

Stephen wanted to see [as LIRG wants to see and as I want to see] a "research in practice" culture. He suggested that this was not about funding (although more funding he agreed would always be welcome) but about policy, frames of reference, outlook, and what he called "positive changes in the professional consensus". And then he wrote something I found very interesting - and a bit scarily prescient.

"For example," suggested Stephen, "if a research enthusiast were to become a chief executive or presidential figure of a major [LIS] organisation, the required catalyst might be evident".

Well now. CILIP has a "research enthusiast" as its CEO - and the British Library has a "research enthusiast" as its CEO - and I begin to detect a renewed enthusiasm for research within Resource. It isn't, of course, down to a few individuals. It requires considerable collective partnership endeavour. But I do believe that the will to engage with that endeavour is in place.

The last few years have been frustrating and difficult for the LIS research community. But our agenda is clear. New opportunities are beginning to emerge. And the longitudinal view with which I began shows that - despite difficulties in the short term - in the long term LIS research does have a powerful and positive impact on LIS practice and service delivery.

CILIP will, as I say, be discussing the research landscape study at the next meeting of our Policy Development Committee. And I look forward to LIRG taking its place at the table as that discussion evolves.

My congratulations to LIRG on becoming part of the CILIP family of Special Interest Groups. And my thanks to you for listening to me this morning.

\section{WORD COUNT: 5935}

